\title{
Oncology in FMUSP and the boundaries between clinical and experimental science
}

\author{
Gilberto de Castro Junior
}

It was 1997 when I decided to be an Oncologist. At that time I was finishing my 2-year training in Clínica Médica at Hospital das Clínicas da Faculdade de Medicina da Universidade de São Paulo - HCFMUSP. Considering all patients admitted to the wards, up to $30 \%$ of them had been diagnosed with a cancer, considering both solid and hematologic neoplasms. The Oncology division of InRad (Instituto de Radiologia) had only six attending physicians in six consulting rooms, shared with Radiotherapy colleagues.

Residency program was started in 1999 with two Oncology fellows in InRad. Nowadays at ICESP (Instituto do Câncer do Estado de São Paulo) we have 14 fellows every year, and our Program of training in Oncology has been ranked among the top 5 best in the annual evaluation of Oncology trainees conducted by the American Society of Clinical Oncology (ASCO) in more than 200 institutions around the world. As the largest Cancer center in South America, in 2014 more than 250,000 outpatient consultations were done at ICESP, and more than 9,000 surgeries.
It is important to mention that research is among the most important activities conducted in our Hospital. ICESP researchers are involved in many research projects from basic science to clinical research, and these results has been presented in international forums and published in high-quality peer-reviewed journals.

One of my areas of interest is Thoracic Oncology, and in the last 15 years I could face a tremendous change in how to better diagnose, treat and prevent lung cancer. Important progress did occur, and some landmarks must be mentioned: (1) incorporation of cisplatin-based adjuvant chemotherapy; (2) better classification of lung cancer in terms of histologic subtypes and more adequate staging; (3) molecular-based selection of systemic therapies; (4) the development of immunotherapy protocols to treat lung cancer, among others.

The intensive participation of our patients in the clinical research program at ICESP allows them to be treated with new treatment options, most of them not available for the cancer patients under SUS coverage. To be part of this initiative is a privilege!

\footnotetext{
Associated Professor of the Oncology Discipline at the Department of the Radiology and Oncolgy, University of Sao Paulo, School of Medicine; Doctor of de Clinical Oncology Service and Chief of the area of Thoracic Oncology and Head and Neck of the Instituto do Câncer do Estado de São Paulo (ICESP).

Mailing address: Instituto do Câncer do Estado de São Paulo, Centro de Oncologia - Pesquisa Clínica. Av. Dr. Arnaldo, 251 - $5^{\circ}$ andar. Cerqueira Cesar. 01246-000 - São Paulo, SP, Brasil. E-mail: gilberto.castro@usp.br.
} 